\title{
A Visual Criterion for Identifying Itô Diffusions as Martingales or Strict Local Martingales
}

\author{
Hardy Hulley and Eckhard Platen
}

\begin{abstract}
It is often important, in applications of stochastic calculus to financial modelling, to know whether a given local martingale is a martingale or a strict local martingale. We address this problem in the context of a time-homogenous diffusion process with a finite lower boundary, presented as the solution of a driftless stochastic differential equation. Our main theorem demonstrates that the question of whether or not this process is a martingale may be decided simply by examining the slope of a certain increasing function. Further results establish the connection between our theorem and other results in the literature, while a number of examples are provided to illustrate the use of our criterion.
\end{abstract}

Mathematics Subject Classification (2000). Primary: 60J60, 60G44; Secondary: 60G40, 60J35, 60J50, 65L99.

Keywords. Diffusions, first-passage times, Laplace transforms, local martingales, ordinary differential equations.

\section{Introduction and main theorem}

The subject of our investigation is a driftless Itô diffusion $X$, taking values in $[l, \infty)$ or $(l, \infty)$, for some $l \in \mathbb{R}$. Given $x>l$, we shall write $\mathrm{P}_{x}$ to denote the probability measure under which this process starts at $x$, and we shall specify its $\mathrm{P}_{x}$-dynamics as follows:

$$
X_{t}=x+\int_{0}^{t} a\left(X_{s}\right) d \beta_{s},
$$

for all $t \geq 0$. Here $\beta$ is a standard scalar Brownian motion, and the measurable function $a$ is assumed to satisfy the following two conditions: (a) $a^{2}(x)>0$, for all $x>l$; and (b) the function $a^{-2}$ is locally integrable. Together, these two conditions ensure that (1.1) possesses a weak solution that is unique in law (see e.g. Karatzas and Shreve [10], Thm. 5.5.15, p. 341). Furthermore, note that $X$ is by construction a $\mathrm{P}_{x}$-local martingale, for all $x>l$, and is therefore also a $\mathrm{P}_{x}$-supermartingale, by 
virtue of being bounded from below. Consequently, the lower boundary $l$ must be absorbing, if it is ever reached.

Local martingales are ubiquitous in stochastic models of financial markets. Firstly, the process that facilitates the transformation from the reference probability measure to a putative equivalent risk-neutral probability measure, for such a model, is only a local martingale, in general. Such a transformation of probability measures only works when the local martingale in question is in fact a martingale. Secondly, even when that process is a martingale, the discounted prices of risky assets are, in general, only local martingales under the associated equivalent risk-neutral probability measure. ${ }^{1}$

A fundamental problem of long-standing importance is to identify conditions for determining whether a given local martingale is in fact a martingale. In this regard, noteworthy sufficient conditions for the case of continuous exponential local martingales have been obtained by Novikov [13] and Kazamaki [11]. Another important line of investigation (see e.g. Azema et al. [2], Galtchouk and Novikov [8], Novikov [14], Elworthy et al. [6, 7] and Takaoka [16]) explored the weak tails of the supremum of a local martingale. This work culminated in a necessary and sufficient condition for classifying an arbitrary continuous local martingale as a martingale or a strict local martingale.

So far only Delbaen and Shirakawa [4] and Kotani [12] appear to have considered explicitly the problem of identifying local martingales of the form (1.1) as martingales or strict local martingales. The former article solves the problem by an application of the first Ray-Knight theorem, while Kotani [12] adopts an analytic approach. Ultimately, these two articles both prove the following theorem: ${ }^{2}$

Theorem 1.1. Given $x>l, X$ is a $\mathrm{P}_{x}$-martingale if and only if

$$
\int^{\infty} \frac{y}{a^{2}(y)} d y=\infty
$$

Proof. See Delbaen and Shirakawa [4], Thm. 1.6 or Kotani [12], Thm. 1.

Given $x>l$, we tackle the problem of determining whether or not $X$ is a $\mathrm{P}_{x}$-martingale differently from the approaches taken by Delbaen and Shirakawa [4] and Kotani [12]. To start with, we derive a necessary and sufficient condition for $X$ to be a $\mathrm{P}_{x}$-martingale that is expressed in terms of its first-passage times. This condition is then translated into the analytic language of diffusions, yielding a striking characterization of martingales within the class of processes described by (1.1).

We begin by briefly recounting some basic facts about time-homogeneous scalar diffusions (the reader is referred to Borodin and Salminen [3], Chap. II for

\footnotetext{
${ }^{1}$ Strictly speaking, this is a simplified version of the true story, since if the asset prices are semimartingales with unbounded jumps, then their discounted values are in general only $\sigma$ martingales under an equivalent risk-neutral probability measure (assuming that one exists).

${ }^{2}$ It must be said, however, that the setting in Kotani [12] is more general than in Delbaen and Shirakawa [4]. For more on this, see the discussion following Proposition 2.2.
} 
more details). The natural starting point is the following linear second-order ODE:

$$
\frac{1}{2} a^{2}(x) u^{\prime \prime}(x)=\alpha u(x),
$$

for all $x>l$ and any fixed $\alpha>0$. This equation has two non-negative linearly independent solutions $\phi_{\alpha}$ and $\psi_{\alpha}$, which may be characterized as the unique (up to multiplicative constant) solutions of (1.2) that are decreasing and increasing, respectively, and which satisfy appropriate boundary conditions, determined by the boundary behaviour of $X$. Both functions are obviously also convex.

To make the connection between (1.1) and (1.2) explicit, let $q$ denote the transition density of $X$ with respect to its speed measure $\mathfrak{m}(d x):=2 a^{-2}(x) d x$. By this we mean

$$
\mathrm{P}_{x}\left(X_{t} \leq z\right)=\mathrm{P}_{x}\left(X_{t}=l\right)+\int_{l}^{z} q(t, x, y) \mathfrak{m}(d y)
$$

for all $x, z>l$. Then we have the following Laplace transform identity: ${ }^{3}$

$$
G_{\alpha}(x, y):=\mathscr{L}_{\alpha}\{q(t, x, y)\}= \begin{cases}w_{\alpha}^{-1} \psi_{\alpha}(x) \phi_{\alpha}(y) & \text { if } x \leq y \\ w_{\alpha}^{-1} \phi_{\alpha}(x) \psi_{\alpha}(y) & \text { if } x \geq y\end{cases}
$$

for all $x, y>l$, where the Wronskian

$$
w_{\alpha}:=\phi_{\alpha}(x) \psi_{\alpha}^{\prime}(x)-\phi_{\alpha}^{\prime}(x) \psi_{\alpha}(x)
$$

is independent of $x$.

The solutions of (1.2) shed further light on (1.1) when we examine the firstpassage times of $X$. To be precise, consider the first-passage time

$$
\tau_{z}:=\inf \left\{t>0 \mid X_{t}=z\right\},
$$

for any $z>l$, and denote its density by $p_{z}$, so that

$$
\mathrm{P}_{x}\left(\tau_{z}<t\right)=\int_{0}^{t} p_{z}(x, s) d s
$$

for all $t \geq 0$. We then obtain the following useful identity:

$$
\mathrm{E}_{x}\left(e^{-\alpha \tau_{z}}\right)=\mathscr{L}_{\alpha}\left\{p_{z}(x, t)\right\}= \begin{cases}\frac{\psi_{\alpha}(x)}{\psi_{\alpha}(z)} & \text { if } x \leq z \\ \frac{\phi_{\alpha}(x)}{\phi_{\alpha}(z)} & \text { if } x \geq z\end{cases}
$$

for all $x, z>l$.

With these preliminaries attended to, we may now formulate and prove the above-mentioned characterization of martingales within the class of processes described by (1.1). The proof relies on the fact that $X$ is a $\mathrm{P}_{x}$-martingale, for any $x>l$, if and only if $\mathrm{E}_{x}\left(X_{t}\right)=x$, for all $t \geq 0$. This follows from the fact that $X$ is a $\mathrm{P}_{x}$-supermartingale, for all $x>l$ :

Theorem 1.2. Given $x>l, X$ is a $\mathrm{P}_{x}$-martingale if and only if

$$
\lim _{z \uparrow \infty} \psi_{\alpha}^{\prime}(z)=\infty \text {. }
$$

\footnotetext{
${ }^{3}$ Note that all Laplace transforms in this paper are computed by integrating over the time domain.
} 
Proof. Choose $z>x$, and note that $X^{\tau_{z}}$ is a (uniformly integrable) $\mathrm{P}_{x}$-martingale. We therefore have

$$
x=\mathrm{E}_{x}\left(X_{t}^{\tau_{z}}\right)=z \mathrm{P}_{x}\left(\tau_{z}<t\right)+\mathrm{E}_{x}\left(\mathbf{1}_{\left\{\tau_{z} \geq t\right\}} X_{t}\right),
$$

for all $t \geq 0$. Since $X$ does not explode (see e.g. Karatzas and Shreve [10], p. 332), it follows that $\lim _{z \uparrow \infty} \mathrm{P}_{x}\left(\tau_{z} \geq t\right)=1$, for all $t \geq 0$. The dominated convergence theorem therefore gives

$$
x-\mathrm{E}_{x}\left(X_{t}\right)=\lim _{z \uparrow \infty} z \mathrm{P}_{x}\left(\tau_{z}<t\right),
$$

for all $t \geq 0$, from which it follows that $X$ is a $\mathrm{P}_{x}$-martingale if and only if

$$
\lim _{z \uparrow \infty} z \mathrm{P}_{x}\left(\tau_{z}<t\right)=0 .
$$

Next, we observe that $X_{\infty}$ exists and satisfies $\mathrm{E}_{x}\left(\left|X_{\infty}\right|\right)<\infty$, by virtue of the fact that $X$ is a $\mathrm{P}_{x}$-supermartingale. We therefore have

$$
0 \leq z \mathrm{P}_{x}\left(\tau_{z}<t\right) \leq z \mathrm{P}_{x}\left(\tau_{z}<\infty\right) \leq|x|+\mathrm{E}_{x}\left(\left|X_{\infty}\right|\right)<\infty,
$$

for all $t \geq 0$, by an application of Doob's maximal inequalities, and we also see that

$$
\mathscr{L}_{\alpha}\left\{|x|+\mathrm{E}_{x}\left(\left|X_{\infty}\right|\right)\right\}=\frac{|x|+\mathrm{E}_{x}\left(\left|X_{\infty}\right|\right)}{\alpha}<\infty,
$$

where $\alpha>0$. We may therefore use the dominated convergence theorem as follows:

$$
\begin{aligned}
\mathscr{L}_{\alpha}\left\{\lim _{z \uparrow \infty} z \mathrm{P}_{x}\left(\tau_{z}<t\right)\right\}=\lim _{z \uparrow \infty} z \mathscr{L}_{\alpha}\left\{\mathrm{P}_{x}\left(\tau_{z}<t\right)\right\} \\
\quad=\lim _{z \uparrow \infty} z \mathscr{L}_{\alpha}\left\{\int_{0}^{t} p_{z}(x, s) d s\right\}=\lim _{z \uparrow \infty} \frac{z}{\alpha} \mathscr{L}_{\alpha}\left\{p_{z}(x, t)\right\}=\lim _{z \uparrow \infty} \frac{z}{\alpha} \frac{\psi_{\alpha}(x)}{\psi_{\alpha}(z)} \\
\quad=\frac{\psi_{\alpha}(x)}{\alpha} \lim _{z \uparrow \infty} \frac{1}{\psi_{\alpha}^{\prime}(z)},
\end{aligned}
$$

where the second-last step follows from (1.4) and the final step is an application of L'Hôpital's rule. The result then follows from (1.5) and (1.6), together with the uniqueness of Laplace transforms.

\section{The connection between Theorems 1.1 and 1.2}

This section formally establishes the correspondence between Theorem 1.1 and Theorem 1.2. In particular, we demonstrate explicitly that the characterization of martingales obtained by Delbaen and Shirakawa [4], Thm. 1.6 or Kotani [12], Thm. 1 may be retrieved from our characterization. There are two steps in this process, the first of which is encapsulated by the following proposition:

Proposition 2.1. Given any $z>l$, we have

$$
\lim _{x \uparrow \infty} \mathrm{E}_{x}\left(\tau_{z}\right) \leq 2 \int_{z}^{\infty}(y-z) \mathfrak{m}(d y) .
$$


Proof. Fix $x>z$, and note that $X^{\tau_{z}}$ is a $\mathrm{P}_{x^{-}}$-supermartingale. It therefore follows that the $\mathrm{P}_{x}$-a.s. limit $X_{\infty}^{\tau_{z}}=X_{\tau_{z}}$ exists and satisfies $\mathrm{E}_{x}\left(\left|X_{\tau_{z}}\right|\right)<\infty$. Next, using Tanaka's formula, we obtain

$$
\left(X_{t}-y\right)^{-}=\left(X_{0}-y\right)^{-}+\underbrace{\int_{0}^{t} \mathbf{1}_{\left\{X_{s} \leq y\right\}} d X_{s}}_{M_{t}}+\frac{1}{2} L_{t}^{y},
$$

for all $t>0$ and $y>l$. Since the process $M$ above is a $\mathrm{P}_{x}$-local martingale with initial value zero, we may infer the existence of an associated localizing sequence of stopping times $\left(\sigma_{n}\right)_{n \in \mathbb{N}}$. Observe that

$$
0 \leq\left(X_{\sigma_{n} \wedge \tau_{z}}-y\right)^{-} \leq(z-y)^{-} \quad \mathrm{P}_{x^{-} \text {-a.s. }},
$$

for all $y>l$ and each $n \in \mathbb{N}$, and recall that the local-time process $L^{y}$ is $\mathrm{P}_{x^{-a} \text {.s. }}$ increasing. Consequently, using the dominated convergence theorem, followed by the optional sampling theorem and the monotone convergence theorem, we get

$$
\begin{aligned}
& \mathrm{E}_{x}\left(\left(X_{\tau_{z}}-y\right)^{-}\right)=\lim _{n \rightarrow \infty} \mathrm{E}_{x}\left(\left(X_{\sigma_{n} \wedge \tau_{z}}-y\right)^{-}\right) \\
& \quad=(x-y)^{-}+\lim _{n \rightarrow \infty} \mathrm{E}_{x}\left(\int_{0}^{\sigma_{n} \wedge \tau_{z}} \mathbf{1}_{\left\{X_{s} \leq y\right\}} d X_{s}\right)+\frac{1}{2} \lim _{n \rightarrow \infty} \mathrm{E}_{x}\left(L_{\sigma_{n} \wedge \tau_{z}}^{y}\right) \\
& \quad=(x-y)^{-}+\frac{1}{2} \mathrm{E}_{x}\left(L_{\tau_{z}}^{y}\right),
\end{aligned}
$$

for all $y>l$. Rearranging this expression, we obtain

$$
\mathrm{E}_{x}\left(L_{\tau_{z}}^{y}\right) \leq 2\left((z-y)^{-}-(x-y)^{-}\right)=2\left((x-z) \wedge(y-z)^{+}\right),
$$

for all $y>l$, since $X_{\tau_{z}} \geq z \mathrm{P}_{x^{-}}$-a.s. Finally, the occupation-measure formula yields

$$
\begin{aligned}
\mathrm{E}_{x}\left(\tau_{z}\right) & =\mathrm{E}_{x}\left(\int_{0}^{\tau_{z}} \mathbf{1}_{\left\{X_{s} \geq z\right\}} d s\right)=\mathrm{E}_{x}\left(\int_{0}^{\infty} \mathbf{1}_{\{y \geq z\}} L_{\tau_{z}}^{y} \mathfrak{m}(d y)\right) \\
& =\int_{z}^{\infty} \mathrm{E}_{x}\left(L_{\tau_{z}}^{y}\right) \mathfrak{m}(d y) \leq 2 \int_{z}^{\infty}(x-z) \wedge(y-z) \mathfrak{m}(d y),
\end{aligned}
$$

and (2.1) follows as a consequence of the monotone convergence theorem.

We next use Proposition 2.1 to obtain the desired correspondence between Theorem 1.1 and Theorem 1.2. In particular, the equivalence between conditions (ii) and (iii) in the following proposition verifies that the criteria in those two theorems are indeed equivalent:

Proposition 2.2. The following conditions are equivalent:

(i) $\phi_{\alpha}(\infty-)>0$;

(ii) $\psi_{\alpha}^{\prime}(\infty-)<\infty$; and

(iii) $\int_{z}^{\infty}(y-z) \mathfrak{m}(d y)<\infty$,

for all $z>l$ and $\alpha>0$. 
Proof. (i) $\Rightarrow$ (ii): Fix $\alpha>0$, and suppose that $\phi_{\alpha}(\infty-)>0$. Since $\phi_{\alpha}$ is decreasing and $\psi_{\alpha}$ is non-negative, we obtain the following inequality from (1.3):

$$
\phi_{\alpha}(x) \psi_{\alpha}^{\prime}(x)=w_{\alpha}+\phi_{\alpha}^{\prime}(x) \psi_{\alpha}(x)<w_{\alpha},
$$

for all $x>l$. Taking limits, it therefore follows that $\psi_{\alpha}^{\prime}(\infty-)<\infty$.

(ii) $\Rightarrow$ (iii): Fix $z>l$ and $\alpha>0$, and suppose that $\psi_{\alpha}^{\prime}(\infty-)<\infty$. Recalling that $\psi_{\alpha}$ is non-negative, increasing and convex, we now obtain

$$
\begin{aligned}
\int_{z}^{\infty} & (y-z) \mathfrak{m}(d y) \leq \int_{z}^{\infty} \frac{\psi_{\alpha}(y)-\psi_{\alpha}(z)}{\psi_{\alpha}^{\prime}(z)} \mathfrak{m}(d y) \leq \frac{1}{\psi_{\alpha}^{\prime}(z)} \int_{z}^{\infty} \psi_{\alpha}(y) \mathfrak{m}(d y) \\
& =\frac{2}{\psi_{\alpha}^{\prime}(z)} \int_{z}^{\infty} \frac{\psi_{\alpha}(y)}{a^{2}(y)} d y=\frac{1}{\alpha \psi_{\alpha}^{\prime}(z)} \int_{z}^{\infty} \psi_{\alpha}^{\prime \prime}(y) d y=\frac{\psi_{\alpha}^{\prime}(\infty-)-\psi_{\alpha}^{\prime}(z)}{\alpha \psi_{\alpha}^{\prime}(z)}<\infty
\end{aligned}
$$

from $\mathfrak{m}(d x):=2 a^{-2}(x) d x$, together with the fact that $\psi_{\alpha}$ satisfies (1.2).

(iii) $\Rightarrow$ (i): Fix $z>l$ and $\alpha>0$, and suppose that $\int_{z}^{\infty}(y-z) \mathfrak{m}(d y)<\infty$. We then obtain

$$
\begin{aligned}
\phi_{\alpha}(\infty-) & =\phi_{\alpha}(z) \lim _{x \uparrow \infty} \mathrm{E}_{x}\left(e^{-\alpha \tau_{z}}\right) \geq \phi_{\alpha}(z) \lim _{x \uparrow \infty} e^{-\alpha \mathrm{E}_{x}\left(\tau_{z}\right)} \\
& \geq \phi_{\alpha}(z) e^{-2 \alpha \int_{z}^{\infty}(y-z) \mathfrak{m}(d y)}>0
\end{aligned}
$$

from (1.4), followed by Jensen's inequality and (2.1).

Theorem 1.1 was first proved by Delbaen and Shirakawa [4], Thm. 1.6, where exactly the same setting was employed as is considered here. However, the result obtained by Kotani [12], Thm. 1 is actually more general than Theorem 1.1, since the local martingales considered there are simply time-homogeneous diffusions in natural scale of which driftless Itô diffusions, such as (1.1), are particular examples. Fortunately, Theorem 1.2 and Propositions 2.1 and 2.2 extend easily to this more general setting (see Hulley [9], Chap. 3 for the details). We are therefore able to recapture Kotani [12], Thm. 1 in full generality.

\section{Some examples}

In this section we examine a number of well-known examples of local martingales of the form (1.1). In each case we compute the fundamental solutions $\phi_{\alpha}$ and $\psi_{\alpha}$ of the ODE (1.2), before using Theorem 1.2 to identify the process as a martingale or a strict local martingale:

Example 3.1 (Squared Bessel process of dimension zero). In this case the statespace is $[0, \infty)$ and $a(x):=2 \sqrt{x}$, for all $x \geq 0$. Solving (1.2) yields

$$
\phi_{\alpha}(x)=\sqrt{x} K_{1}(\sqrt{2 \alpha x}) \quad \text { and } \quad \psi_{\alpha}(x)=\sqrt{x} I_{1}(\sqrt{2 \alpha x}),
$$

for all $x \geq 0$ and $\alpha>0$ (see Figure 1). Here $I_{1}$ and $K_{1}$ are modified Bessel functions of the first and second kinds, respectively (see e.g. Abramowitz and Stegun [1], 

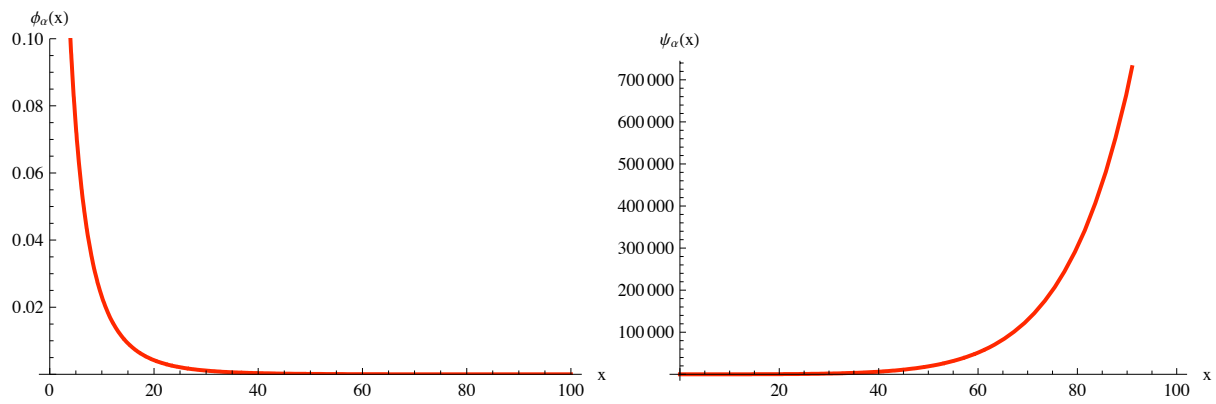

Figure 1. The functions $\phi_{\alpha}$ and $\psi_{\alpha}$ for the squared Bessel process of dimension zero.
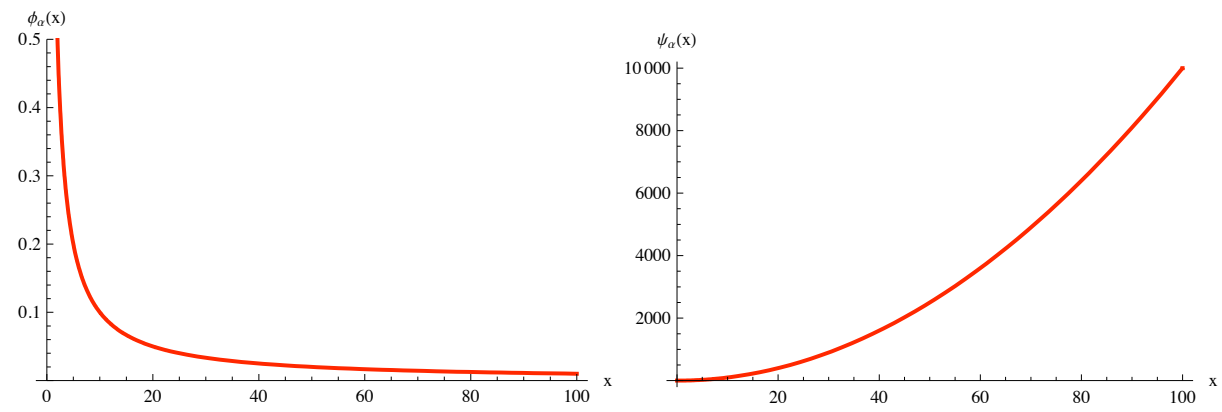

Figure 2. The functions $\phi_{\alpha}$ and $\psi_{\alpha}$ for driftless geometric Brownian motion.

Chap. 9). It now follows from the recurrence relations for modified Bessel functions in Abramowitz and Stegun [1], Eqns. (9.6.26) that

$$
\psi_{\alpha}^{\prime}(x)=\frac{1}{2 \sqrt{x}} I_{1}(\sqrt{2 \alpha x})+\frac{\sqrt{\alpha}}{2 \sqrt{2}}\left(I_{0}(\sqrt{2 \alpha x})+I_{2}(\sqrt{2 \alpha x})\right),
$$

for all $x \geq 0$ and $\alpha>0$, and we obtain $\psi_{\alpha}^{\prime}(\infty-)=\infty$. We may therefore deduce that $X$ is a martingale, by Theorem 1.2 .

Example 3.2 (Driftless geometric Brownian motion). In this case the state-space is $(0, \infty)$ and $a(x):=x$, for all $x>0$. Solving (1.2) yields

$$
\phi_{\alpha}(x)=x^{-\frac{1}{2}(\sqrt{8 \alpha+1}-1)} \quad \text { and } \quad \psi_{\alpha}(x)=x^{\frac{1}{2}(\sqrt{8 \alpha+1}+1)} \text {, }
$$

for all $x>0$ and $\alpha>0$ (see Figure 2). It is easily seen that $\psi_{\alpha}^{\prime}(\infty-)=\infty$, for all $\alpha>0$, from which we may deduce that $X$ is a martingale, by Theorem 1.2. 

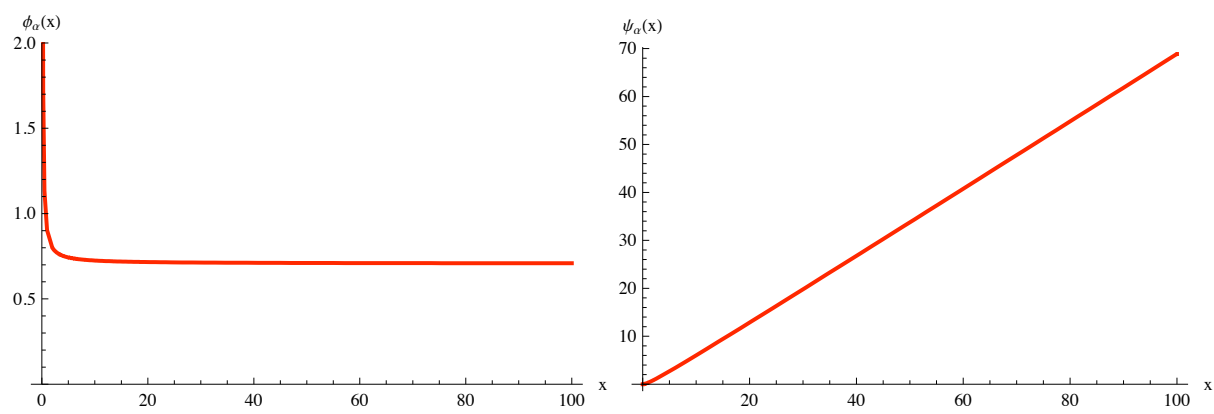

Figure 3. The functions $\phi_{\alpha}$ and $\psi_{\alpha}$ for the inverted squared Bessel process of dimension four.

Example 3.3 (Inverted squared Bessel process of dimension four). In this case the state-space is $(0, \infty)$ and $a(x):=2 x^{\frac{3}{2}}$, for all $x>0$. Solving (1.2) then yields

$$
\phi_{\alpha}(x)=\sqrt{x} I_{1}\left(\sqrt{\frac{2 \alpha}{x}}\right) \quad \text { and } \quad \psi_{\alpha}(x)=\sqrt{x} K_{1}\left(\sqrt{\frac{2 \alpha}{x}}\right),
$$

for all $x>0$ and $\alpha>0$ (see Figure 3). It now follows from the recurrence relations for modified Bessel functions in Abramowitz and Stegun [1], Eqns. (9.6.26) that

$$
\psi_{\alpha}^{\prime}(x)=\frac{1}{2 \sqrt{x}} K_{1}\left(\sqrt{\frac{2 \alpha}{x}}\right)+\frac{\sqrt{\alpha}}{2 \sqrt{2} x}\left(K_{0}\left(\sqrt{\frac{2 \alpha}{x}}\right)+K_{2}\left(\sqrt{\frac{2 \alpha}{x}}\right)\right),
$$

for all $x>0$ and $\alpha>0$, and we obtain $\psi_{\alpha}^{\prime}(\infty-)=\frac{1}{\sqrt{2 \alpha}}$. We may therefore deduce that $X$ is a strict local martingale, by Theorem 1.2 .

Example 3.4 (Inverted Bessel process of dimension three). In this case the statespace is $(0, \infty)$ and $a(x):=x^{2}$, for all $x>0$. Solving (1.2) then yields

$$
\phi_{\alpha}(x)=x\left(e^{\frac{\sqrt{2 \alpha}}{x}}-e^{-\frac{\sqrt{2 \alpha}}{x}}\right) \quad \text { and } \quad \psi_{\alpha}(x)=x e^{-\frac{\sqrt{2 \alpha}}{x}},
$$

for all $x>0$ and $\alpha>0$ (see Figure 4). It is easily seen that $\psi_{\alpha}^{\prime}(\infty-)=1$, for all $\alpha>0$, from which we may deduce that $X$ is a strict local martingale, by Theorem 1.2.

Based on the examples above, it seems natural to speculate that $X$ is a $\mathrm{P}_{x^{-}}$ martingale if and only if its diffusion coefficient is asymptotically sub-linear, in the sense that $\lim _{x \uparrow \infty} \frac{a(x)}{x}<\infty$. The following example from Ekström and Tysk [5] (who analyze it differently) is therefore quite surprising:

Example 3.5 (Kummer's local martingale). In this example the state-space is $(1, \infty)$ and we set $a(x):=x \sqrt{\ln x}$, for all $x>1$. In order to solve the ODE (1.2), 

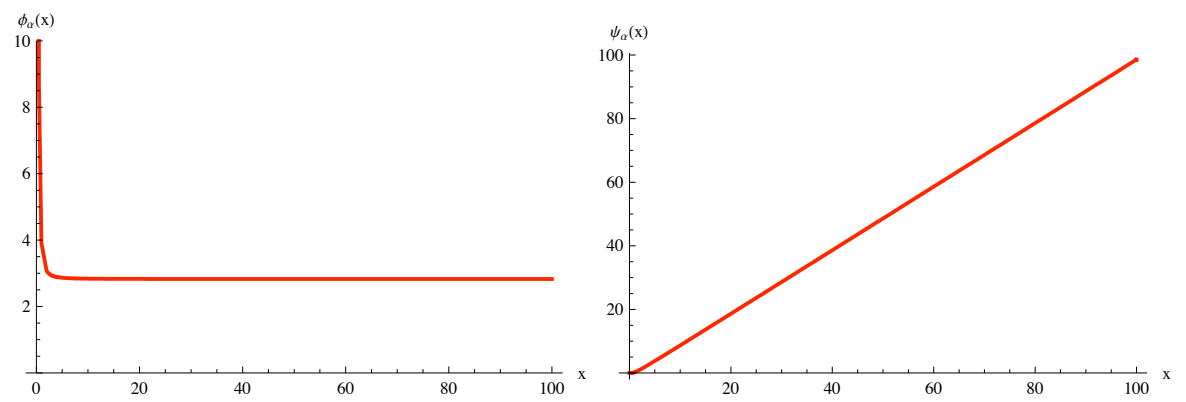

FiguRE 4. The functions $\phi_{\alpha}$ and $\psi_{\alpha}$ for the inverted Bessel process of dimension three.
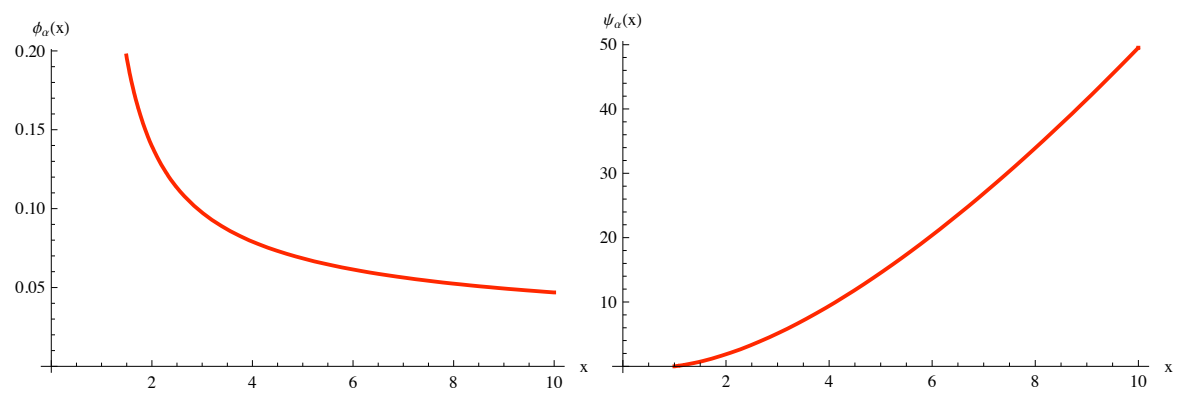

Figure 5. The functions $\phi_{\alpha}$ and $\psi_{\alpha}$ for Kummer's local martingale.

we first employ the transformation of variables $\ln x \mapsto \xi$, and set $v_{\alpha}(\xi):=u_{\alpha}(x)$, for all $x>1$ and $\alpha>0$. Equation (1.2) then becomes

$$
\xi v_{\alpha}^{\prime \prime}(\xi)-\xi v_{\alpha}^{\prime}(\xi)-2 \alpha v_{\alpha}(\xi)=0,
$$

for all $\xi>0$ and $\alpha>0$. This is recognizable as an instance of Kummer's equationalso known as a degenerate hypergeometric equation (see e.g. Polyanin and Zaitsev [15], pp. 137-139) — whose solutions may be expressed in terms of the confluent hypergeometric functions $M$ and $U$ (see e.g. Abramowitz and Stegun [1], Chap. 13). For our original equation (1.2), we then obtain

$$
\phi_{\alpha}(x)=\ln x U(1+2 \alpha, 2, \ln x) \quad \text { and } \quad \psi_{\alpha}(x)=\ln x M(1+2 \alpha, 2, \ln x),
$$

for all $x>1$ and $\alpha>0$ (see Figure 5). It now follows from Abramowitz and Stegun [1], Eqn. (13.4.8) that

$$
\psi_{\alpha}^{\prime}(x)=\frac{1}{x} M(1+2 \alpha, 2, \ln x)+\frac{(1+2 \alpha) \ln x}{2 x} M(2+2 \alpha, 3, \ln x),
$$

for all $x>1$ and $\alpha>0$, and we obtain $\psi_{\alpha}^{\prime}(\infty-)=\infty$. We may therefore deduce that $X$ is a martingale, by Theorem 1.2 . 


\section{Acknowledgments}

Thanks to Kristoffer Glover for numerous insights concerning the solution of ordinary differential equations, and to the anonymous referee for several helpful suggestions for improving the overall structure of the paper.

\section{References}

[1] M. Abramowitz and I. A. Stegun, editors, Handbook of Mathematical Functions, Dover, 1972.

[2] J. Azema, R. F. Gundy, and M. Yor, Sur l'integrabilité uniforme des martingales continues, In: Séminaire de Probabilités XIV, Lecture Notes in Mathematics, 784 (1980), 53-61, Springer, Berlin.

[3] A. N. Borodin and P. Salminen, Handbook of Brownian Motion, Birkhäuser, Basel, second edition, 2002.

[4] F. Delbaen and H. Shirakawa, No arbitrage condition for positive diffusion price processes, Asia-Pacific Finan. Markets, 9 (3-4) (2002), 159-168.

[5] E. Ekström and J. Tysk, Bubbles, convexity and the Black-Scholes equation, 2008.

[6] K. D. Elworthy, X. M. Li, and M. Yor, On the tails of the supremum and the quadratic variation of stricly local martingales, In: Séminaire de Probabilités XXXI, Lecture Notes in Mathematics, 1655 (1997), 113-125, Springer, Berlin.

[7] K. D. Elworthy, X.-M. Li, and M. Yor, The importance of strictly local martingales; applications to radial Ornstein-Uhlenbeck processes, Probab. Theory Related Fields, 115 (1999), 325-355.

[8] L. I. Galtchouk and A. A. Novikov, On Wald's equation. Discrete time case, In: Séminaire de Probabilités XXXI, Lecture Notes in Mathematics, 1655 (1997), 126-135, Springer, Berlin.

[9] H. Hulley, Strict Local Martingales in Continuous Financial Market Models, PhD thesis, 2009.

[10] I. Karatzas and S. E. Shreve, Brownian Motion and Stochastic Calculus, Springer, New York, second edition, 1991.

[11] N. Kazamaki, On a problem of Girsanov, Tohōku Math. J., 29 (4) (1977), $597-600$.

[12] S. Kotani, On a condition that one-dimensional diffusion processes are martingales, In: Séminaire de Probabilités XXXIX, Lecture Notes in Mathematics, 1874 (2006), 149-156, Springer, Berlin.

[13] A. A. Novikov, On an identity for stochastic integrals, Theory Probab. Appl., 17 (4) (1972), 717-720.

[14] A. A. Novikov, Martingales, Tauberian theorem, and strategies of gambling, Theory Probab. Appl., 41 (4) (1997), 716-729.

[15] A. D. Polyanin and V. F. Zaitsev, Handbook of Exact Solutions for Ordinary Differential Equations, CRC Press, Boca Raton, 1995. 
[16] K. Takaoka, Some remarks on the uniform integrability of continuous martingales, In: Séminaire de Probabilités XXXIII, Lecture Notes in Mathematics, 1709 (1999), 327-333, Springer, Berlin.

\section{Hardy Hulley}

School of Finance and Economics

University of Technology, Sydney

P.O. Box 123

Broadway, NSW 2007

Australia

e-mail: hardy.hulley@uts.edu.au

Eckhard Platen

School of Finance and Economics \& Department of Mathematical Sciences

University of Technology, Sydney

P.O. Box 123

Broadway, NSW 2007

Australia

e-mail: eckhard.platen@uts.edu.au 